\title{
Integrating system biology tools to decipher the mechanisms of action of herbal medicine
}

\begin{abstract}
The advance of bioinformatics tools aims at unraveling the molecular mechanisms of herbal medicine and contributing to better health treatments for human conditions. The science and practice of herbal medicine is emerging around the world to develop better compounds used for drug design, but mainly to better understand the mechanistic design of herb formulas used in the long history for treating various medical purposes. The research race of developing an ideal systematic approach would gain much popularity and confidence among health professionals that can recommend these products to their patients. The challenge resides in deciphering what approach and parameters to use for clearly demonstrating the efficient role of herbal formulas. In the past 5-10 years ago, researchers attempted investigating the influence of herbal formula on gene expression profiling which let other groups develop larger sets of transcriptomes and predictive interactions. Examining changes in metabolites is valuable as it provides relevant information associated with clinical observations. To demonstrate the therapeutic effects of herbal formulas, researchers developed in silico models to provide predictive pharmacological data that can validate the in vitro and in vivo studies.
\end{abstract}

Keywords: herbal medicine, gene expression, protein-protein interaction, signaling pathways, network analysis
Volume 7 Issue I - 2018

\author{
Benoit Leclerc, ${ }^{1,2}$ Priscilla $\mathrm{Wu}^{2}$ \\ 'MatrixHerbs Research Institute, Canada \\ ${ }^{2}$ MatrixHerbs Biotechnology Inc, Canada
}

Correspondence: Benoit Leclerc, Matrix Herbs Research Institute, Unit I-223 Bank Street, Ottawa, Ontario, Canada,Tel 16139838|85, Email matrixherbsbiotechnology@gmail.com

Received: January 29, 2018 | Published: February 06, 2018
Abbreviations: TCM, traditional chinese medicine; MCF-7, breast cancer cell line; HUVEC, human umbilical vascular endothelial cells; Nrf2, nuclear factor erythroid 2-related factor; ENA-78, epithelial cells derived neutrophil activating peptide; KEAP1, kelchlike ech-associated protein 1; miRNA, micro RNA

\section{Introduction}

Since herbal medicine had been employed long ago in the treatment of various human health conditions especially in Asia countries, the Western world has recently recognized its medical benefits. Still, the therapeutic index of herbal medicine remains unknown, and their corresponding human clinical studies are largely undetermined. Many health professionals make a reservation using them for patient's treatment without previous rigorous efficacy and safety studies which prevent herbal products approval under healthcare practices. The Food and Drug Administration had approved two botanical drugs, to named Veregen in 2006 and Fulyzaq in 2012 while other formulas are in clinical trial phases $2 \& 3$. The main hurdle many scientists around the world face is "demystifying" by demonstrating the mechanistic concept of herbal formulas to restore the biological processes from abnormal to health status in a variety of disorders/diseases. Thereof, this article aims at presenting the capabilities of current biology tools and their integration into a bioinformatics platform to dissect the system mechanisms by which human cells used after being exposed to herbal formula treatment.

\section{Microarray analysis}

A great number of herbal medicine studies have demonstrated positive treatment effects in human health conditions. According to the Figure 1 polynomial trendline, the last 10-year publications database has contributed to an important increase of scientific interest towards examining the influence of herbal medicine on human gene expression. In the earliest period, scientists discovered gene expression profiling associated with herbal formula. Notably, Vigconic VI-28 used to activate the immune system of patients with chronic HIV and hepatitis B infections was reported to differentially modulate gene expression in particular T lymphocytes for increasing interleukin 1, growth-related protein beta, and epithelial cells derived neutrophil activating peptide (ENA-78) revealed on DNA microarray analysis. ${ }^{1}$ A company developed Circulat to prevent necrotic plantar foot manifestation associated with type 2 diabetes. Following Affymetrix oligonucleotide microarrays analysis, among 187 genes identified on the human fibroblast cell line MRC-5, interleukin 6, HMGA1, SLC19A2 \& C4A have been associated with the development of diabetes. $^{2}$

In the second period, scientists strove into developing bioinformatics approaches to discover new targets from the molecular network modulated following treatment with their herbal medicine formula. For example, Si Wu Tang formula, composed of four herbs, has been recognized for treating gynecological diseases. To provide insights of the complex therapeutic effects of traditional Chinese medicine, a human breast cancer cell line (MCF-7) quantitated by microarray and real-time PCR to show differentially expressed genes. $^{3}$ The authors identified the up-regulation of HMOX1, GCLC, GCLM, SLC7A11 and NQO1 in a dose-dependent manner associated with the activation of nuclear factor erythroid 2-related factor 2 (Nrf2) cytoprotective pathway. The connectivity map database of withaferin A and resveratrol-treated MCF-7 cells revealed highly similar gene expression profiling to $\mathrm{Si} \mathrm{Wu}$ Tang treatment suggesting its uses as a nontoxic chemopreventive agent. ${ }^{3}$ Also, the connectivity map of estradiol-treated MCF-2 cells matched with gene expression profile of $\mathrm{Si} \mathrm{Wu}$ Tang treatment, indicating its phytoestrogenic activity. ${ }^{3}$ Further experimental analyses confirmed the estrogenic potency of $\mathrm{Si}$ $\mathrm{Wu}$ Tang using estrogen luciferase reporter assay, pointing to MCF- 
7 cells growth stimulation at low concentration which was blocked by the addition of estrogen receptor antagonist, ${ }^{4}$ and to support the formula clinical use for cancer prevention. Another group uses the traditional Chinese medicine PHY906 with the chemotherapy CTP-11 to induce significantly pro-inflammatory and pro-apoptotic transcription factors such as IRF5, IFNAR2 \& IRF-1 which allows the immune-mediated tumor apoptosis following its administration to female BDF-1 mice carrying subcutaneous colon tumors. ${ }^{5}$ Since the process of angiogenesis is vital in diabetic wound healing, a team of researchers investigated the role of NF3-treated human umbilical vein endothelial cells (HUVEC) in a time-regulated manner. Following microarray analysis in NF3-treated human umbilical vascular endothelial cells (HUVEC), gene expression profiling at $6 \mathrm{~h}$ shifted at $16 \mathrm{~h}$. For example, genes were involved in inflammatory (MAP3K14 vs. IL1RAPL2 \& NR1H4), tumorigenicity (SLC5A8 vs. miR28), cell proliferation (BIRC5, EPCAM, INSL4, MMP8 \& NPR3 vs. PRL \& TFAP2A), cell migration (CXCR7, EPCAM, HAND1 \& MMP8 vs. miR28, PRL, SCG2), tubular formation (CXCR7, EPCAM \& MMP8 vs. PRL) and angiogenesis signaling (BIRC5, CXCR7, EPCAM, HAND1, MMP8 \& UBD vs. miR28, NR1H4 \& PRL) ${ }^{6}$

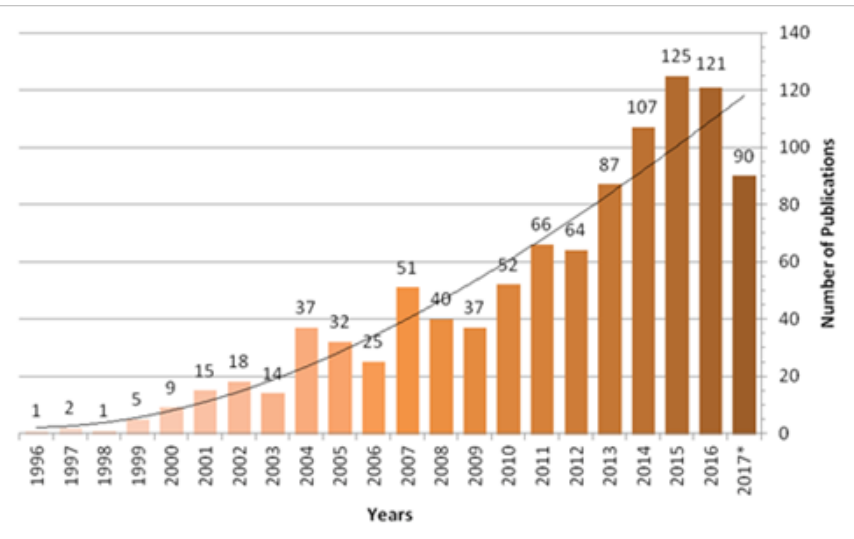

Figure I Total number of PubMed publications that investigated the impact of herbal medicine on human gene expression ( $N=999)$. *The lower number of publications observed in this year could be due to journals publishing time. As well, yearly fluctuation in publications number is influenced by the type and amount of research grants awarded.

Recently, Bupleurum \& Tang Kuei Formula (Xiao Yao Wan), used to treat posttraumatic stress disorder, has shown significant reduction of endogenous and exogenous reactive oxidative stress level in both human glioblastoma T98G and neuroblastoma SH-SY5Y cell lines. ${ }^{7}$ In addition, the microarray analysis showed activation of the NRF2mediated oxidative stress response by inhibiting its interaction with Kelch-like ECH-associated protein 1 (KEAP1) which had high affinity with paeoniflorin and baicalin according to the in silico analysis. This increased the antioxidant effect of heme oxygenase-1 protein expression (alias HMOX1) responsible to protect against psychiatric diseases. Thus, incorporating microarray data with herbal target database would infer signaling pathway enrichment of the predicted target of TCM as well constructing an intelligent network of herbal ingredients-targets-drugs actions would provide insights about the mechanistic of the herbal formula [8]. On the other hands, to elaborate the potential pharmacological mechanisms of $\mathrm{Si} \mathrm{Wu}$ Tang formula, 20 predicted targets were identified by interconnecting known targets in TCM database and differentially expressed genes. ${ }^{8}$ Since herbal medicine bind to multiple tissues' targets, encoded by differential expressed genes, their therapeutic efficacy is consequently enhanced. ${ }^{9,10}$ According to the types of herbal combinations, mutual counteractions and mutual suppressions between compounds reduce significantly the overall formula's side effects.

\section{Transcriptomics analysis}

Microarray provides an excellent tool to evaluate global gene expression profiling in cell lines-treated herbal formula. To provide more tangible findings, transcriptomics can analyze multiple transcriptional events simultaneously using gene expression profiling data integrated into biological interaction database validated by molecular techniques. This approach assesses the multi-component activity of polyherbal remedy to dissect its mechanistic found in human conditions is needed. For example, Padma 28 has been shown to possess high antioxidative effect in human hepatoma HepG2 cells due to its inducing effect on the antioxidant response element reporter gene expression and repressive effect on intracellular reactive oxidative stress levels related to differential expression of genes quantified by microarray and real-time PCR. Interconnecting differential genes with pathways, molecular interactions networks and biological processes most perturbed in HepG2 cells revealed the cells transcriptional signatures which were time and dosage dependent-Padma 28 treatments, such as the many targets associated with TNF and INF gamma in the cardiovascular system development $\&$ function, cell-to-cell signaling \& interaction and inflammatory responses networks. ${ }^{10} \mathrm{~A}$ randomized, double-blind trial reported the identification of human miRNA-18a-5p, miRNA-326, miRNA-1182 \& miRNA-193b-5p as potential biomarkers to improve the diagnostic accuracy of Fuzheng-Huayu formula in treating patients with hepatitis B-caused cirrhosis. ${ }^{11}$ The differentially expressed miRNA and predicted targets, obtained from databases, were combined to build miRNA target network by using the Cluster ONE algorithm. The combination of the four miRNAs was associated with 12 KEGG pathways and was identified as potential biomarkers.

\section{Proteomics analysis}

Fewer studies have looked over protein expression profiling to underlie the signaling pathways and regulatory networks following TCM treatment. To compare the differential proteins expression of HUVEC after the Chinese 2-herb formula (NF3) treatment in both static and scratch conditions, a database for annotation, visualization and integrated discovery tool was employed to generate comprehensive molecular functions driving the proangiogenic response of wound healing in diabetic foot ulcer. ${ }^{12}$ To further analyze the interconnectivity between signaling pathways vs. protein-protein interaction vs. biological processes between static and scratch conditions, Genomatrix Pathway System was employed to provide insights of the regulatory mechanisms elicit by NF3 to modulate wound healing angiogenesis, such as the down-regulation of plasminogen activator inhibitor-1 and up-regulation of Annexin A2 confirmed by proliferation and migration assay. The authors also identified proteins involved in cytoskeleton regulation, transcription \& translation, cellcell interaction and ROS defense in HUVEC-treated NF3. ${ }^{12}$ Another research group investigated the hepatic anti-fibrotic mechanisms of the SRE formula in an experimental rat model present most of the clinical manifestations as found in human liver diseases. The counteracted effect of the SRE formula on DMN-induced hepatotoxicity indicated repression of alpha-smooth muscle actin, PCNA expression and collagen content in the liver as well as serum levels in amino acid enzymes. Among 1,500 protein spots on 2D-PAGE (proteomics) analysis, repressed cytoskeleton proteins and enzymes involved in oxidative stress were identified as catechol O-methyltransferase, 
3-tubulin and K8. To assign the differential expressed proteins on 2D and peptide mass fingerprint to biological networks to the relevant anti-fibrotic mechanisms-related pathways induced by SRE formula, Gene GO MetaCore analysis identified hypoxia and oxidative stress \& cytoskeleton intermediate filaments having the most significant p-value to the GO annotation. As predicted by network analysis, induction of glutathione peroxide, superoxide dismutase and catalase expressed in the liver by SRE was dose-dependent manner, as SRE up-regulated Nrf-2 which is a major transcription factor involved in the antioxidant process to protect the liver damage. ${ }^{13}$

\section{Metabolomics analysis}

One approach to providing valuable information for clinical diagnosis and TCM intervention is to elucidate the relationship between metabolites changes and biological (pathological/ physiological) pathways. For instances, the diagnosis and Xiang$\mathrm{Fu}-\mathrm{Si}-\mathrm{Wu}$ formula treatment for primary dysmenorrhea could be improved by measuring endogenous metabolites in patients' blood plasma and urine samples. In a cross-sectional study, UPLC coupled with QTOF/MS and QqQ/MS techniques were utilized to quantify levels of lysophosphatidylcholine 16:1, 20:4, 18:2, 16:0, $18: 1,10: 0$, estrone, 17-hydroxyprogesterone, myristoylglycine \& palmitoylglycine all induced significantly in primary dysmenorrhea while phytosphingosine, dihydrocortisol \& sphingosine were all repressed significantly compared to healthy subjects. These metabolic perturbations participate in glycerophospholipid metabolism, sphingolipid metabolism and steroid hormone biosynthesis. Metabolic deviations in patients' plasma and urine samples restore progressively to the normal level from one to three consecutive menstrual cycles XFSWF intervention. These metabolites could be considered as potential biomarkers for the primary dysmenorrhea as well as targets for XFSWF bioactive compounds and should be correlated with the neural-endocrine-immune pathways to aid in the development of clinical diagnosis methods. ${ }^{14}$ Similar methodologies evaluating metabolites perturbations associated with depression and polycystic ovary syndrome which were restored to normal levels after TCM intervention has been reported in human ${ }^{15,16}$ and dysmenorrhea, menopausal metabolic syndrome and gut microbiota dysbiosis in rats. ${ }^{17-19}$ Further research studies are required to evaluate the therapeutic effect of formula's chemical compounds towards metabolic biomarkers found in plasma or urine driven human diseases. Therefore, identifying TCM chemical compounds by metabolomics is based on fingerprinting analysis of tissues treated by different TCM extracts at different retention times having known biological activity.

\section{Systems pharmacology models}

Following metabolomics analysis, the approach consists of using systems biology tools to provide further evidence regarding the therapeutic effect(s) of TCM acting on multiple physiological levels in the treatment of human sub-health status or multiple pathological stages to reduce cancerous cells growth. Systems pharmacology models are based on the synergistic interactions between compounds, targets and pathways to probe the therapeutic efficiency of the overall TCM recipe. As exemplified, Compound Danshen formula, used in the treatment of cardiovascular diseases, was modeling by in silico approaches, such as assessing compounds-target associations, compound-pathway connections and disease-target interactions and all of these networks were analyzed by two plugins to generate enriched biological networks. ${ }^{20}$ Genes and proteins were associated with vasculature diseases; then Compound Danshen formula's candidate compounds were assigned to renin-angiotensin-aldosterone, glucocorticoid and inflammatory pathway, PPAR signaling, TGFbeta, L-arginine/NO signaling \& platelet aggregation pathway; finally, the formula's target compounds were linked to cardiovascular disease in association with other diseases. ${ }^{20}$ Uncovering candidate compounds, molecular targets and interaction network involved in Xiao-Chaihu and Da-Chaihu decoction would allow examining their recipe optimization as well designing new TCM formulas with ideal therapeutic effects. ${ }^{21}$ In another herbal medicine application, Diesun Miaofang has been employed for the treatment of traumatic injury. To shed light on the pharmacological properties of the formula, a systems pharmacology model was built by combining cluster ligands (i.e. affinity), human intestinal absorption and aqueous solution prediction (i.e. drug-likeness properties), chemical mapping (i.e. structure-activity-relationship), molecular docking (i.e. compounds to target proteins involved in blood circulation and anti-inflammation) and network pharmacology (i.e. potential active compound to targets). Results showed similar (parallel) profile distribution between key topological properties of compound-target network associated with activating blood and removing stasis mechanism with compoundtarget network promoting qi circulation and relieving pain mechanism; for instances ferulic acid showed strong interaction with catecholO-methyl transferase, phenylethanolamine N-methyltransferase, phenyl-ethanolamine, phosphodiesterase $5 \mathrm{~A}$ in homeostasis \& blood circulation and with factor-IXa in anti-inflammation. ${ }^{22} \mathrm{~A}$ third application of systems pharmacology integrated pharmacokinetic parameters, target fishing and network analysis to demonstrate the extend compounds-target interactions support clinical effects of Tianshu formula in treating migraine. Results showed that Rhizoma chuanxiong modulates PTGS2, ESR1, NOS2, HTR1B \& NOS3 targets acting on the vascular and nervous systems and secondary to the inflammation and pain-related pathways while the adjuvant Gastrodia elata acts on ABAT, HTR1D, ALOX15 \& KCND3 to alleviate migraine symptoms, such as vomiting, vertigo and gastrointestinal disorders. ${ }^{23}$ Lately, danggui-shaoyao-san decoction has been recognized as an herbal therapy for neurodegenerative diseases. Using oral bioavailability screening, drug-likeness, target identification and compound-target \& compound-target-disease network analyses, $10 \%$ of predicted candidate bioactive compounds may represent the mechanisms of action of the formula. ${ }^{24}$ Similar systems pharmacology approaches on herbal medicine have been developed for cardiovascular diseases, vitiligo, mental disorders and psoriasis. $^{25-28}$

\section{Conclusion}

Substantial findings related to the mechanistic of herbal medicine at the transcriptomics, proteomics, metabolomics and systems biology tools indicate worldwide scientific interest in the field. To decode the principles of herbal formulation in treating effectively one or several diseases, one should build predictive models linking potential candidate compounds to target to pathways to diseases and confirming the in silico network analysis by developing human cell culture studies and molecular assays. An inclusive compounds analytical model should be developed to examine at the molecular level the efficiency of herbal formulas in the treatment of diseases. Future trends should further explore the epigenetics influence of herbal medicine related to the mechanisms of histone modulation specific to diseases. For instances, the inherited genetic variation specific to the human population will undoubtedly influence the treatment success rate, so the need to develop pharmacogenomics studies is indispensable. 


\section{Acknowledgements}

None.

\section{Conflict of interest}

The author declares no conflict of interest.

\section{References}

1. Pan Hammarstrom Q, Wen S, Hammarstrom L. Cytokine gene expression profiles in human lymphocytes induced by a formula of traditional Chinese medicine, vigconic VI-28. J Interferon Cytokine Res. 2006;26(9):628-636.

2. Antoshechkin A, Olalde J, Magarici M, et al. Analysis of effects of the herbal preparation Circulat on gene expression levels in cultured human fibroblasts. Phytother Res. 2007;21(8):777-789.

3. Wen Z, Wang Z, Wang S, et al. Discovery of molecular mechanisms of traditional Chinese medicinal formula $\mathrm{Si}-\mathrm{Wu}$-Tang using gene expression microarray and connectivity map. PLoS One. 2011;6(3):e18278.

4. Liu M, Fan J, Wang S, et al. Transcriptional profiling of Chinese medicinal formula $\mathrm{Si}-\mathrm{Wu}-\mathrm{Tang}$ on breast cancer cells reveals phytoestrogenic activity. BMC Complement Altern Med. 2013;13:11.

5. Wang E, Bussom S, Chen J, et al. Interaction of a traditional Chinese Medicine (PHY906) and CPT-11 on the inflammatory process in the tumor microenvironment. BMC Med Genomics. 2011;4:38.

6. Tam JC, Ko CH, Koon CM, et al. Identification of Target Genes Involved in Wound Healing Angiogenesis of Endothelial Cells with the Treatment of a Chinese 2-Herb Formula. PLoS One. 2015;10(10):e0139342.

7. Hong C, Cao J, Wu CF, et al. The Chinese herbal formula Free and Easy Wanderer ameliorates oxidative stress through KEAP1-NRF2/HO-1 pathway. Sci Rep. 2017;7(1):11551.

8. Fang Z, Lu B, Liu M, et al. Evaluating the pharmacological mechanism of Chinese medicine $\mathrm{Si}-\mathrm{Wu}-$ Tang through multi-level data integration. PLoS One. 2013;8(11):e72334.

9. Panossian A, Seo EJ, Wikman G, et al. Synergy assessment of fixed combinations of Herba Andrographidis and Radix Eleutherococci extracts by transcriptome-wide microarray profiling. Phytomedicine. 2015;22(11):981-992.

10. Klein A, Wrulich OA, Jenny M, et al. Pathway-focused bioassays and transcriptome analysis contribute to a better activity monitoring of complex herbal remedies. BMC Genomics. 2013;14:133.

11. Chen Q, Wu F, Wang M, et al. Transcriptional Profiling and miRNATarget Network Analysis Identify Potential Biomarkers for Efficacy Evaluation of Fuzheng-Huayu Formula-Treated Hepatitis B Caused Liver Cirrhosis. Int J Mol Sci. 2016;17(6):E883.

12. Tam JC, Ko CH, Zhang $\mathrm{C}$, et al. Comprehensive proteomic analysis of a Chinese 2-herb formula (Astragali Radix and Rehmanniae Radix) on mature endothelial cells. Proteomics. 2014;14(17-18):2089-2103.

13. Pan TL, Wang PW, Huang CH, et al. Herbal formula, Scutellariae radix and Rhei rhizoma attenuate dimethylnitrosamine-induced liver fibrosis in a rat model. Sci Rep. 2015;5:11734.
14. Liu P, Duan J, Wang P, et al. Biomarkers of primary dysmenorrhea and herbal formula intervention:an exploratory metabonomics study of blood plasma and urine. Mol Biosyst. 2013;9(1):77-87.

15. Tian JS, Peng GJ, Gao XX, et al. Dynamic analysis of the endogenous metabolites in depressed patients treated with TCM formula Xiaoyaosan using urinary (1)H NMR-based metabolomics. J Ethnopharmacol. 2014;158:1-10.

16. Lu C, Zhao X, Li Y, et al. Serum metabolomics study of Traditional Chinese medicine formula intervention to polycystic ovary syndrome. $J$ Pharm Biomed Anal. 2016;120:127-133.

17. Huang X, Su S, Duan JA, et al. Effects and mechanisms of Shaofu-Zhuyu decoction and its major bioactive component for Cold - Stagnation and Blood - Stasis primary dysmenorrhea rats. J Ethnopharmacol. 2016;186:234-243.

18. Wan XM, Zhang M, Zhang P, et al. Jiawei Erzhiwan improves menopausal metabolic syndrome by enhancing insulin secretion in pancreatic $\beta$ cells. Chin J Nat Med. 2016;14(11):823-834.

19. Yu M, Jia HM, Zhou C, et al. Urinary and Fecal Metabonomics Study of the Protective Effect of Chaihu-Shu-Gan-San on Antibiotic-Induced Gut Microbiota Dysbiosis in Rats. Sci Rep. 2017;7:46551.

20. Li X, Xu X, Wang J, et al. A system-level investigation into the mechanisms of Chinese Traditional Medicine:Compound Danshen Formula for cardiovascular disease treatment. PLoS One. 2012;7(9):e43918.

21. Li B, Tao W, Zheng C, et al. Systems pharmacology-based approach for dissecting the addition and subtraction theory of traditional Chinese medicine: An example using Xiao-Chaihu-Decoction and Da-ChaihuDecoction. Comput Biol Med. 2014;53:19-29.

22. Zheng CS, Fu CL, Pan CB, et al. Deciphering the underlying mechanisms of Diesun Miaofang in traumatic injury from a systems pharmacology perspective. Mol Med Rep. 2015;12(2):1769-1776.

23. Li Y, Zhang J, Zhang L, et al. Systems pharmacology to decipher the combinational anti-migraine effects of Tianshu formula. $J$ Ethnopharmacol. 2015;174:45-56.

24. Luo Y, Wang Q, Zhang Y. A systems pharmacology approach to decipher the mechanism of danggui-shaoyao-san decoction for the treatment of neurodegenerative diseases. J Ethnopharmacol. 2016;178:66-81.

25. Liu J, Mu J, Zheng C, et al. Systems-Pharmacology Dissection of Traditional Chinese Medicine Compound Saffron Formula Reveals Multi-scale Treatment Strategy for Cardiovascular Diseases. Sci Rep. 2016;6:19809.

26. Pei $\mathrm{T}$, Zheng $\mathrm{C}$, Huang $\mathrm{C}$, et al. Systematic understanding the mechanisms of vitiligo pathogenesis and its treatment by Qubaibabuqi formula. $J$ Ethnopharmacol. 2016;190:272-287.

27. Shen X, Zhao Z, Luo X, et al. Systems Pharmacology Based Study of the Molecular Mechanism of SiNiSan Formula for Application in Nervous and Mental Diseases. Evid Based Complement Alternat Med. 2016;2016:9146378

28. Sundarrajan S, Arumugam M. A systems pharmacology perspective to decipher the mechanism of action of Parangichakkai chooranam, a Siddha formulation for the treatment of psoriasis. Biomed Pharmacother. 2017;88:74-86. 